\title{
Spray droplet modeling 2. An interactive Eulerian-Lagrangian model of evaporating spray droplets
}

\author{
J. B. Edson \\ Woods Hole Oceanographic Institution, Woods Hole, Massachusetts \\ S. Anquetin \\ Laboratoire des Ecoulements Géophysiques et Industriels, Université Joseph Fourrier, Grenoble, France \\ P. G. Mestayer and J. F. Sini \\ Laboratoire de Mécanique des Fluides, Ecole Centrale de Nantes, Nantes, France
}

\begin{abstract}
This paper describes an interactive Eulerian-Lagrangian model of the turbulent transport of evaporating droplets. A $k-\varepsilon$ (where $k$ is turbulent kinetic energy and $\varepsilon$ is its rate of dissipation) turbulence closure model is used to accurately simulate stable, near-neutral, and unstable boundary layers within the large air-sea interaction tunnel at the Institut de Mécanique Statistique de la Turbulence (IMST), Luminy, France. These results are then used with the Lagrangian model described in part 1 [Edson and Fairall, 1994]. The coupled model is shown to give excellent agreement with droplet dispersion measurements made during the 1988 Couche Limite Unidimensionelle Stationnaire d'Embrums (CLUSE, a French acronym that translates to one-dimensional stationary droplet boundary layer) campaign. Additionally, this paper describes how the coupled model can now be used to investigate the interaction between the evaporating droplets and the turbulent fields of temperature and humidity. The investigation shows that although the influence of the droplets is small under the conditions simulated at IMST, the potential for substantial modification of the surface energy budget exists for high-wind conditions over the ocean.
\end{abstract}

\section{Introduction}

This paper describes an interactive Eulerian-Lagrangian model of the turbulent transport of evaporating spray droplets. The model has been developed to address some of the limitations described by Edson and Fairall [1994] (hereinafter referred to as part 1), and to allow the use of the model in more complicated flows. The model development involved the integration of the Lagrangian model described in part 1 with an Eulerian model of turbulent flows that uses prognostic equations for the evolution of the turbulent kinetic energy $k$ and its rate of dissipation $\epsilon$. The integrated code has been christened Gwaihir, and we shall refer to the model as such in the following discussion.

The initial tests of the $k-\epsilon$ model are conducted through simulations of developing boundary layers using a two-dimensional version of the code. The model runs are initialized and

Copyright 1996 by the American Geophysical Union.

Paper number $95 \mathrm{JC} 03280$.

0148-0227/96/95JC-03280 $\$ 5.00$ compared with measurements taken within the large air-water interaction simulation tunnel at the Institut de Mécanique Statistique de la Turbulence (IMST), Luminy, France, during the 1988 Couche Limite Unidimensionelle Stationnaire d'Embrums (CLUSE, a French acronym that translates to onedimensional stationary droplet boundary layer) campaign [Mestayer et al., 1990]. These simulations have provided a means to test the various droplet dispersion aspects of Gwaihir, as well as the performance of the Eulerian code in simulations of the marine atmospheric surface layer.

The paper describes in some detail both the physical model and the numerical procedure used in our approach. It also addresses some of the advantages of this combined (Eulerian plus Lagrangian) approach over separate approaches (Eulerian or Lagrangian) in simulations of the turbulent transport of heavy particles. It then concludes with the results from the interactive model for simulations of droplet dispersion in both a laboratory and marine atmospheric surface layer.

\section{Eulerian $k-\epsilon$ Model}

The Eulerian code used in this simulation is derived from a $k-\epsilon$ model developed at the Laboratoire de Mécanique des Fluids, 
Ecole Centrale de Nantes, France, to simulate flows around urban structures [Lévi Alvarès et al., 1990; Lévi Alvarès and Sini, 1992; Lakehal et al., 1996]. In the atmospheric surface layer, expressions for the instantaneous velocity field for incompressible fluid flow can be written

$$
\begin{gathered}
\frac{\partial U_{j}}{\partial x_{j}}=0 \\
\frac{\partial U_{i}}{\partial t}+U_{j} \frac{\partial U_{i}}{\partial x_{j}}=-\frac{1}{\rho_{a}} \frac{\partial P}{\partial x_{i}}-g_{i} \frac{\boldsymbol{\Theta}_{v}-\Theta_{v}^{r}}{\Theta_{v}^{r}}+v \frac{\partial^{2} U_{i}}{\partial x_{j}^{2}}
\end{gathered}
$$

where Einstein's summation notation is used and the Boussinesq approximation has been applied, $v$ is the kinematic viscosity; $\Theta_{v}$ is the ambient virtual potential temperature; $g_{i}=(0,0,-g)$ where $g$ is the gravitational acceleration; and $\rho_{a}$ and $\Theta_{\nu}^{\prime}$ and virtual potential temperature of the reference state of the fluid, respectively [Landahl and Mollo-Christensen, 1986]. In (2) the pressure field $P$ represents the departure from the reference pressure field in hydrostatic balance. In developing equations designed to study flows where the mean departure from hydrostatic equilibrium can be nonzero (e.g., around a building), Sini [1986] and Sini and Dekeyser [1987] decomposed this departure from hydrostatic equilibrium into mean and fluctuating parts. The Reynolds averaged equations for the mean variables are then given by

$$
\begin{gathered}
\frac{\partial \bar{U}_{j}}{\partial x_{j}}=0 \\
\frac{\partial \bar{U}_{i}}{\partial t}+\bar{U}_{\bar{j}} \frac{\partial \bar{U}_{i}}{\partial x_{j}}= \\
-\frac{1}{\overline{\rho_{a}}} \frac{\partial \bar{P}}{\partial x_{i}}-g_{i} \frac{\overline{\boldsymbol{\Theta}_{v}}-\overline{\boldsymbol{\Theta}_{v}^{r}}}{\overline{\boldsymbol{\Theta}_{v}^{r}}}-\frac{\partial \overline{u_{i} u_{j}}}{\partial x_{j}}+v \frac{\partial^{2} \bar{U}_{i}}{\partial x_{j}^{2}} \\
\frac{\partial \overline{\boldsymbol{\theta}}}{\partial t}+\overline{U_{j}} \frac{\partial \overline{\boldsymbol{\theta}}}{\partial x_{j}}=\frac{\partial \overline{u_{j} \theta}}{\partial x_{j}}+\frac{S_{H}}{\rho_{a} c_{p}} \\
\frac{\partial \bar{Q}}{\partial t}+\bar{U}_{j} \frac{\partial \bar{Q}}{\partial x_{j}}=\frac{\partial \overline{u_{j}}}{\partial x_{j}}+S_{q}
\end{gathered}
$$

where the overbar represents an ensemble average; lower case letters denote the turbulent fluctuations; the total specific humidity $Q$ has since been added to allow for the inclusion of moisture in the model equations; $c_{p}$ is the specific heat at constant pressure; and $S_{H}$ and $S_{q}$ represent source terms for sensible heat and moisture, respectively. The source/sink terms are discussed in detail below.

\subsection{Closure}

The Reynolds-stress tensor is modeled using the Boussinesq eddy diffusivity concept

$$
-\bar{u}_{i} u_{j}=v_{T}\left[\frac{\partial \bar{U}_{i}}{\partial x_{j}}+\frac{\partial \bar{U}_{j}}{\partial x_{i}}\right]-\frac{2}{3} \delta_{i j} k
$$

where $\delta_{i j}$ is the Krönecker delta tensor, $v_{T}$ is the eddy diffusivity, and $k$ is the turbulent kinetic energy (TKE) defined as

$$
k=\frac{1}{2} \overline{u_{j} u_{j}}
$$

Similarly, the scalar fluxes are modeled using

$$
\begin{aligned}
& -\overline{u_{j} \theta}=K_{\theta} \frac{\partial \bar{\Theta}}{\partial x_{j}} \\
& -\overline{u_{j} q}=K_{q} \frac{\partial \bar{Q}}{\partial x_{j}}
\end{aligned}
$$

where $K_{\theta}$ and $K_{q}$ are the exchange coefficients for potential temperature and specific humidity, respectively. The exchange coefficients are parameterized as

$$
\begin{aligned}
& v_{T}=C_{\mu} \frac{k^{2}}{\epsilon} \\
& K_{\theta}=P r_{T} v_{T} \\
& K_{q}=S c_{T} v_{T}
\end{aligned}
$$

where $C_{\mathrm{p}}$ is a model coefficient and the Prandtl and Schmidt numbers for turbulent diffusion are assigned the same value, $\operatorname{Pr}_{T}=S c_{T}=0.95$ [Högström, 1988].

Closure is then accomplished through prognostic equations for both the turbulent kinetic energy and its rate of dissipation

$$
\begin{gathered}
\frac{\partial k}{\partial t}+\bar{U}_{j} \frac{\partial k}{\partial x_{j}}= \\
-\overline{u_{i} u_{j}} \frac{\partial \bar{U}_{i}}{\partial x_{j}}-\frac{g_{j}}{\boldsymbol{\theta}_{v}} \overline{u_{j} \theta_{v}}+\frac{\partial}{\partial x_{j}}\left[\frac{v_{T}}{\sigma_{k}} \frac{\partial k}{\partial x_{j}}\right]-\epsilon \\
\frac{\partial \epsilon}{\partial t}+\bar{U}_{j} \frac{\partial \epsilon}{\partial x_{j}}=-C_{\epsilon 1} \frac{\epsilon}{k}\left[\overline{u_{i} u_{j}} \frac{\partial \bar{U}_{i}}{\partial x_{j}}+\frac{g_{j}}{\boldsymbol{\theta}_{v}} \overline{u_{j} \theta_{v}}\right] \\
+\frac{\partial}{\partial x_{j}}\left[\frac{v_{T}}{\sigma_{\epsilon}} \frac{\partial \epsilon}{\partial x_{j}}\right]-C_{\epsilon 2} \frac{\epsilon^{2}}{k}
\end{gathered}
$$


where $\epsilon$ is the rate of irreversible dissipation of kinetic energy into thermal energy and $\sigma_{k}, C_{\epsilon 1}, \sigma_{\epsilon}$, and $C_{\epsilon 2}$ are model coefficients. By parameterizing the transfer coefficients in this fashion, we believe that the diffusive properties of the flow are more representative of the intensity $k$ and persistence $k / \epsilon$ of the turbulence than could be obtained through the normal application of first-order closure (i.e., $K$-theory). The two-equation approach is also less complicated and less expensive to run than higher-order models, while still maintaining many of their advantages over those of first order or those that assume a balance between production and dissipation of TKE.

\subsection{Model Coefficients}

The numerical coefficients are chosen such that they are representative of the laboratory boundary layer (LBL) at IMST. The values of the coefficients $\sigma_{\epsilon}, C_{\epsilon 1}$, and $C_{\epsilon 2}$ are based on semiempirical relationships [e.g., Avva et al., 1988; Duynkerke, 1988]. These relationships have been shown to be in good agreement with observation made within the air-sea interaction tunnel at IMST [Mestayer, 1980]. In particular, the near-surface dissipation rate is well defined in neutral conditions by

$$
\epsilon=\frac{u_{*}^{3}}{\kappa z}
$$

where $u_{\text {. }}$ is the velocity scaling parameter (or more commonly the friction velocity) and the von Karman's constant, $\kappa$, is assigned a value of 0.4 [Högström, 1988].

We use the measurements of Mestayer [1980] to relate the friction velocity to vertical velocity variance as $\sigma_{w}^{2}=1.69 u_{*}^{2}$. The constant of proportionality $C_{\mu}$ used in (11) to define the eddy diffusivity, is assigned the value 0.09 . This value has been successfully used to simulate a number of laboratory flows [e.g., Launder and Spalding, 1974]. The value of $C_{\epsilon 2}$ is found from measurements taken in homogeneous grid turbulence where the diffusion and production of TKE are negligible. This leads to a situation where there exists a balance between the advection and dissipation of TKE such that $C_{\mathrm{\epsilon} 2}$ becomes the only constant of significance in (14) and (15). Values of $C_{\epsilon 2}$ determined from these measurements are found to lie within the range of 1.8 to 2.0 [Avva et al., 1988]; we have chosen the commonly used value of 1.92 for the present model. In highly stratified flows, where the Richardson number has reached its critical value $R i_{c}$, the transport processes again become negligible and (14) and (15) can be combined to give [Duynkerke, 1988]

$$
C_{\epsilon 1}=C_{\epsilon 2}\left(1-R i_{c}\right)
$$

Additionally, near the surface, where we expect a balance between mechanical production and dissipation of TKE, a logarithmic velocity profile, and negligible transport, (15) reduces to

$$
C_{\epsilon 1}=C_{\epsilon 2}-\frac{\kappa^{2}}{\sigma_{\epsilon} C_{\mu}^{1 / 2}}
$$

Table 1. Numerical Constants Used in the Model Simulations

\begin{tabular}{ll}
\hline Constant & Value \\
\hline$C_{\mu}$ & 0.09 \\
$C_{\epsilon 1}$ & 1.44 \\
$C_{\epsilon 2}$ & 1.92 \\
$\sigma_{k}$ & 1.00 \\
$\sigma_{\epsilon}$ & 1.11 \\
$\kappa$ & 0.40 \\
$P r_{T}$ & 0.95 \\
$S c_{T}$ & 0.95 \\
\hline$S e{ }^{2}$ &
\end{tabular}

See section 2.1 for variable definitions.

Equations (17) and (18) can then be combined to obtain

$$
\sigma_{\epsilon}=\frac{\kappa^{2}}{C_{\mu}^{1 / 2} C_{\epsilon 2} R i_{c}}
$$

In the present model we have assigned the values 1.0 and 0.25 to $\sigma_{k}$ and $R i_{c}$, respectively. Using these values and the above expressions we obtain the values of the coefficients listed in Table 1.

\subsection{Numerical Formulation}

The numerical formulation is an adaptation of the code Chensi developed by Lévi Alvarès [1992] and Lévi Alvares and Sini [1992] for an inhomogeneous, three-dimensional (3D) grid. The solution of the system of equations is found using the Marker-and-Call computing method as presented by Hirt and Harlow [1967]. The numerical model utilizes a staggered grid configuration that defines the velocity components at the cell faces and the scalar variables at the cell centers as shown in Figure 1. Variable grid spacing is used to allow for smaller grid increments in regions where strong gradients are expected, in an effort to reduce the numerical noise in the algorithm. The derivatives are determined with a second-order, finite volume scheme that takes into account the variable grid spacing as described by Lévi Alvarès [1992].

The numerical method is explicit in time and uses an upwind weighted difference scheme for the advection terms and a centered difference scheme for the diffusion terms. The continuity equation is satisfied for the mean velocities at each time step through use of the artificial compressibility method described by Chorin [1967]. The system of equations is then marched forward in time until the desired level of convergence is reached such that the Eulerian variables represent the steady state solution. In the current model we assume that a steady state has been reached when changes in model parameters between successive time steps approach the computer's 


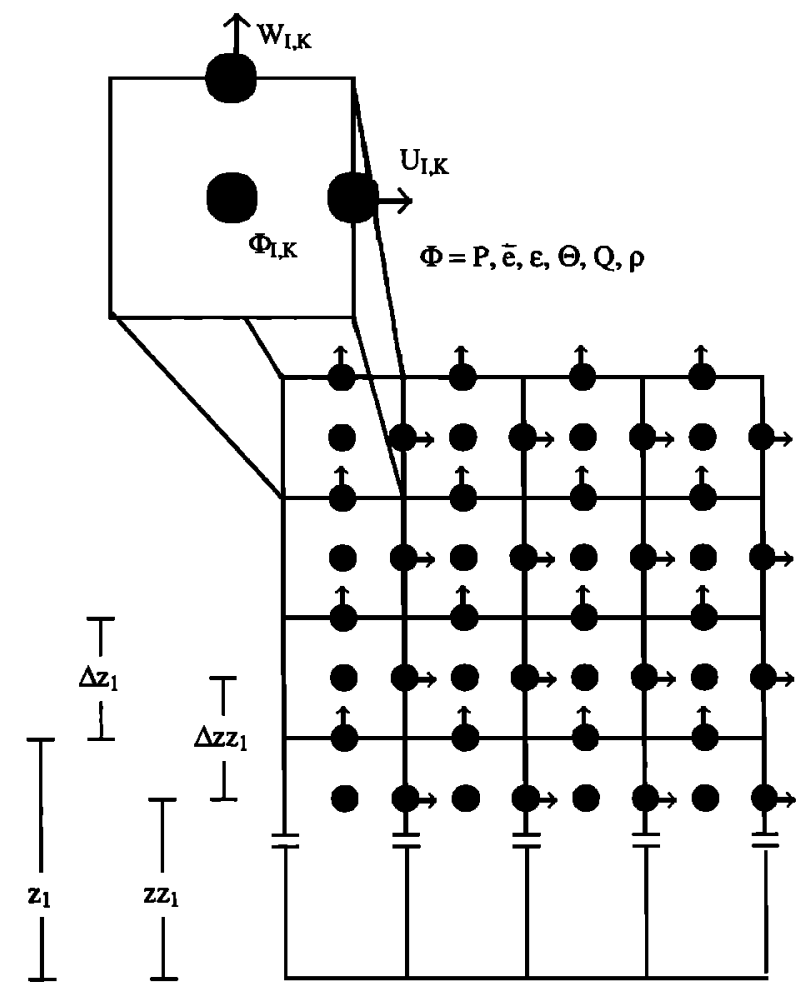

Figure 1. The staggered grid configuration used in the Eulerian model.

numerical precision (currently, a Sun Sparcstation 2 using double precision).

In the simulations that follow the height of the lowest grid point is set to $1 \mathrm{~cm}$. Therefore it is safe to assume that molecular effects can be neglected since this height is at least an order of magnitude larger than the roughness length $z_{0}$. The 2-D domain of the model simulations measures $50 \mathrm{~m}$ by $0.85 \mathrm{~m}$, which is roughly the length and height, respectively, of the turbulent boundary layer at IMST. The determination of the boundary conditions using the configuration shown in Figure 1 is discussed in some detail below.

\subsection{The Boundary Conditions}

In the $k-\epsilon$ code we define the grid as shown in Figure 1, where the parameters in the first grid are not defined at its center. This approach saves computer time and improves the determination of the derivatives at the lowest grid points. It also allows us to move the height of the first grid point to a region well above the viscous sublayer such that we can safely ignore molecular effects. However, these noncentered grid points found in the first cells require special consideration. The velocity component parallel to the surface (in these simulations the horizontal velocity) is found using the wall law given by Launder and Spalding [1974] to estimate the surface stress

$$
u_{*}^{2}=\frac{\tau_{o}}{\rho_{a}}=\frac{\kappa\left(\overline{U_{2}}-\overline{U_{o}}\right) C_{\mu}^{1 / 4} k^{1 / 2}}{\ln \left(\frac{z z_{2}}{z_{o}}\right)}
$$

where the subscripts $o$ and 2 represent the values at $z_{o}$ and at the second grid point, respectively. Equation (20) reduces to the classic logarithmic profile when a constant stress layer prevails near the surface; that is, we assume that

$$
u_{*}=C_{\mu}^{1 / 4} k^{1 / 2}
$$

The roughness length at the end of the tunnel is estimated from

$$
\left.z_{o}\right|_{I_{\max }}=\frac{v}{C_{z} C_{\mu}^{1 / 4} k_{I_{\max }, 2}^{1 / 2}}+\frac{C_{g} C_{\mu}^{1 / 2} k_{I_{\max }, 2}}{g}
$$

where the $I_{\max }$ denotes the value at the outflow section of the model domain. The first term on the right-hand side of (22) gives the roughness length for a smooth surface with $C_{z}=9$, while the second term is based on Charnock's formula [Charnock, 1955] using $C_{g}=0.017$ [Garratt, 1977]. The value of $z_{o}$ at the lowest grid point in the inflow section is assumed to equal that for a smooth surface. Using these two values, we then assume that the surface roughness increases in a linear fashion with fetch. This assumption is consistent with actual measurements made within the tunnel.

The vertical velocity at the lowest grid points is then determined by requiring that the continuity equation is satisfied in the lowest cells. First, the average horizontal velocity through each grid face $U_{I}$ is found by integrating the $\log$ profile from $z_{o}$ to $z_{1}$. The vertical velocity is then found using (3), with $W_{o}=$ 0 , such that

$$
W_{1}=-\frac{U_{I}-U_{I-1}}{\Delta x}\left(z_{1}-z_{o}\right)
$$

where $\Delta x$ is the width of each cell.

A condition of zero diffusive flux of TKE is used between the first two grid points

$$
\left(\frac{\partial k}{\partial z}\right)_{1,2}=0
$$

The dissipation rate of TKE is found by assuming that the mechanical production of TKE is equal to dissipation in the near-wall region. This leads to a relationship between the TKE and its dissipation rate at the lowest grid point given by

$$
\epsilon_{1}=\frac{\left(C_{\mu}^{1 / 4} k^{1 / 2}\right)^{\beta}}{\kappa z z_{1}}
$$

The horizontal velocity profile at the upstream boundary (hereafter referred to as the entrance) is fixed using a profile based on the measurements made by Selva [1979] in the IMST tunnel using a Pitot tube and highly accurate manometer. Neumann conditions (i.e., zero gradient) are used for all variables at the upper and downstream boundaries, except for the vertical velocity at the upper boundary, which is set equal to zero. 
The initial values of $k$ are found by inversion of (21), while initial values of $\epsilon$ are assigned using (16). The value of $u_{*}$ used in both relationships is assigned an initial value equal to two fifths the value of $u$. measured at a distance (fetch) of 30 $\mathrm{m}$ from the tunnel entrance. The inflow values of $k$ and $\epsilon$ are then allowed to adjust through the use of Neumann conditions. The temperature and specific humidity profiles at the tunnel entrance are fixed using the value measured at $0.75 \mathrm{~m}$, except for at the lowest grid point that is given the surface value. This is consistent with both measurements and the action of the heat exchangers in the tunnel, which act to hold the air temperature and dew point constant, mix the air thoroughly, and break down any undesirable eddies. At the lower boundary the temperature is given the value of the water surface temperature $T_{w}$, while the specific humidity is assigned its saturation value at $T_{w}$.

\subsection{Eulerian Model Results}

The Eulerian model's velocity results are in good agreement with the measurements made using a Pitot tube during the CLUSE campaign as shown in Figure 2. The various curves in Figure 2 depict the evolution of the wind profile at various fetches. The velocity profile measured at $30 \mathrm{~m}$ gives excellent agreement with the model profile at a fetch of $29 \mathrm{~m}$. This result indicates that the velocity evolution is accurately modeled, especially since there is some uncertainty as to where we define the fetch to be equal to 0 . The small discrepancy between the model and measurements at the top of the boundary layer is the result of the confluence of the tunnel's two boundary layers.

In general, the vertical structure of temperature and humidity along the length of the tunnel cannot be adequately described

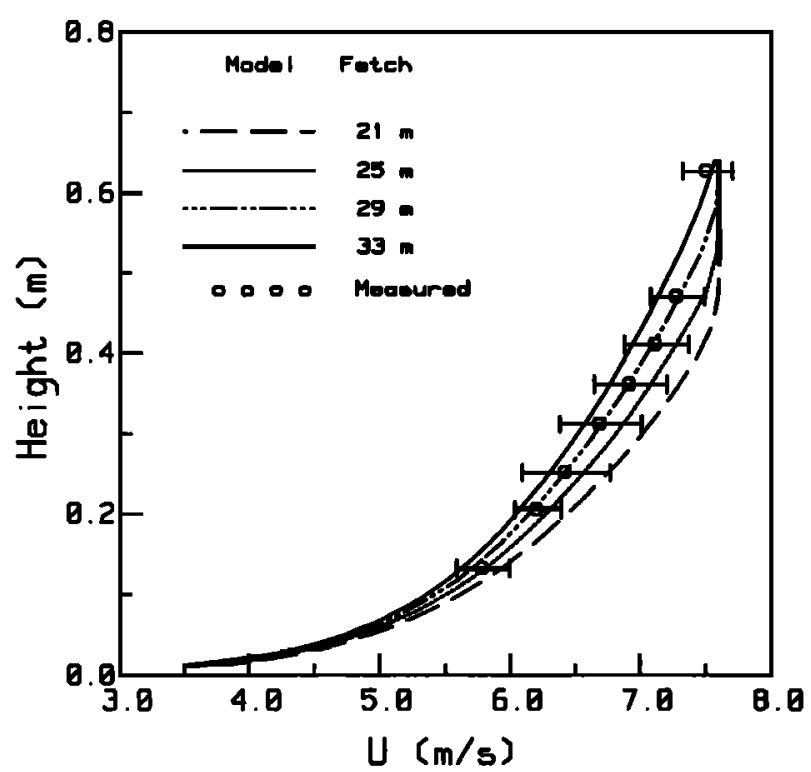

Figure 2. A comparison of the velocity measurements made at a fetch of $30 \mathrm{~m}$ in the Institut de Mechanique Statistique de la Turbulence (IMST) tunnel with the Eulerian model results. The group of curves depicts the model-derived wind profiles at the fetches indicated. The error bars denote the standard deviation of the measured velocity variance.

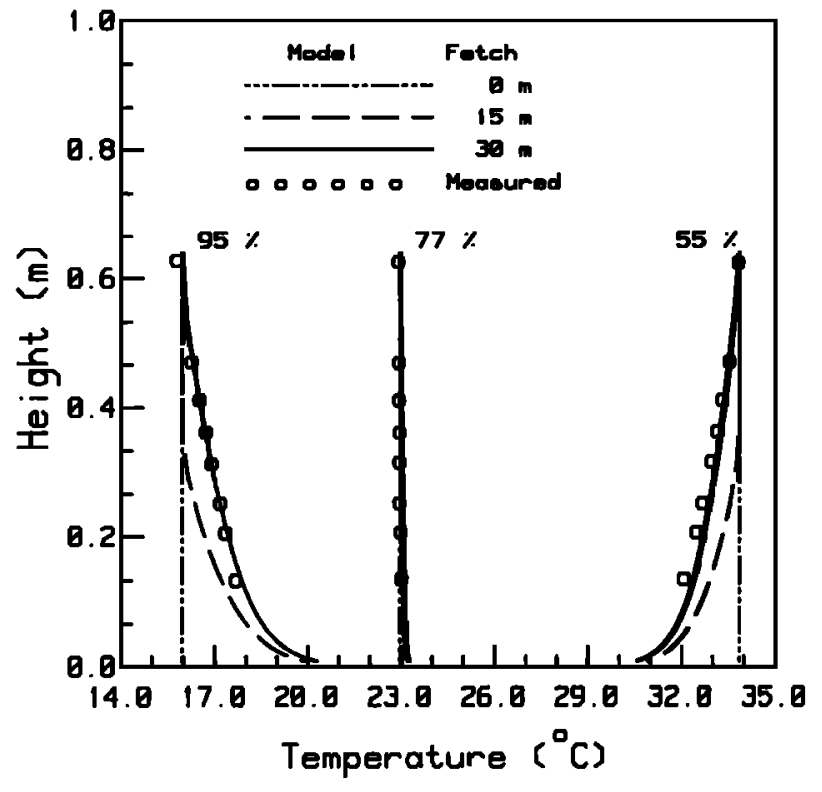

Figure 3. A comparison of the temperature measurements made at a fetch of $30 \mathrm{~m}$ in the IMST tunnel with the Eulerian model results. The three sets of data are representative of the high-, medium-, and low- humidity $(95 \%, 77 \%$, and $55 \%$, respectively) runs made during CLUSE. The nominal wind speed in all three cases is approximately $7.5 \mathrm{~m} \mathrm{~s}^{-1}$. The group of curves depicts the model-derived temperature profiles at the fetches indicated. The standard deviation of the measured temperature variance is given at each level for the medium humidity run.

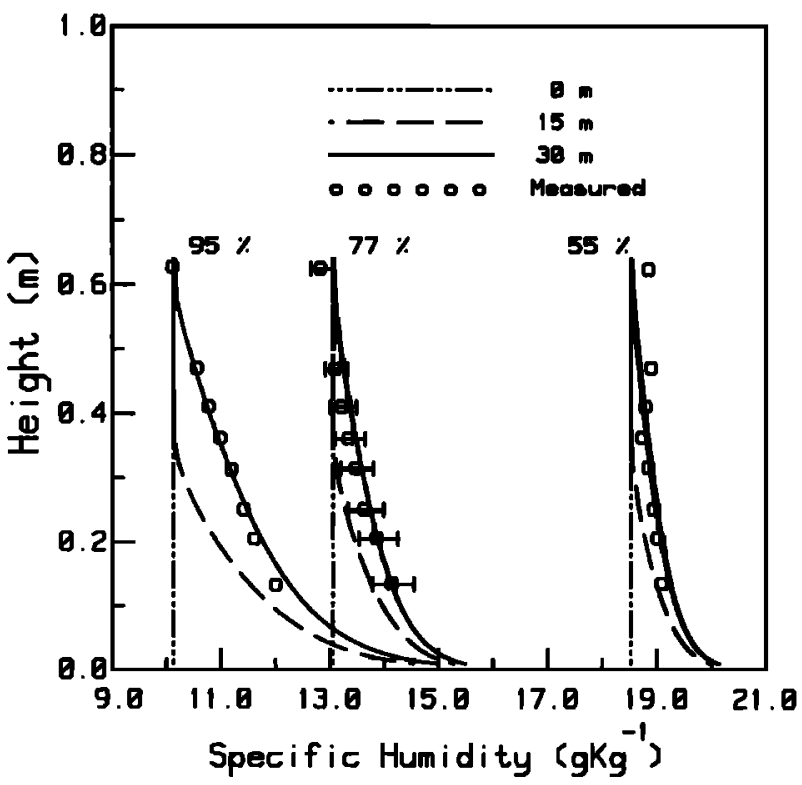

Figure 4. A comparison of the specific humidity measurements made at a fetch of $30 \mathrm{~m}$ in the IMST tunnel with the Eulerian model results. The conditions are the same as in Figure 3. The standard deviation of the measured specific humidity variance is given at each level for the medium humidity run. 


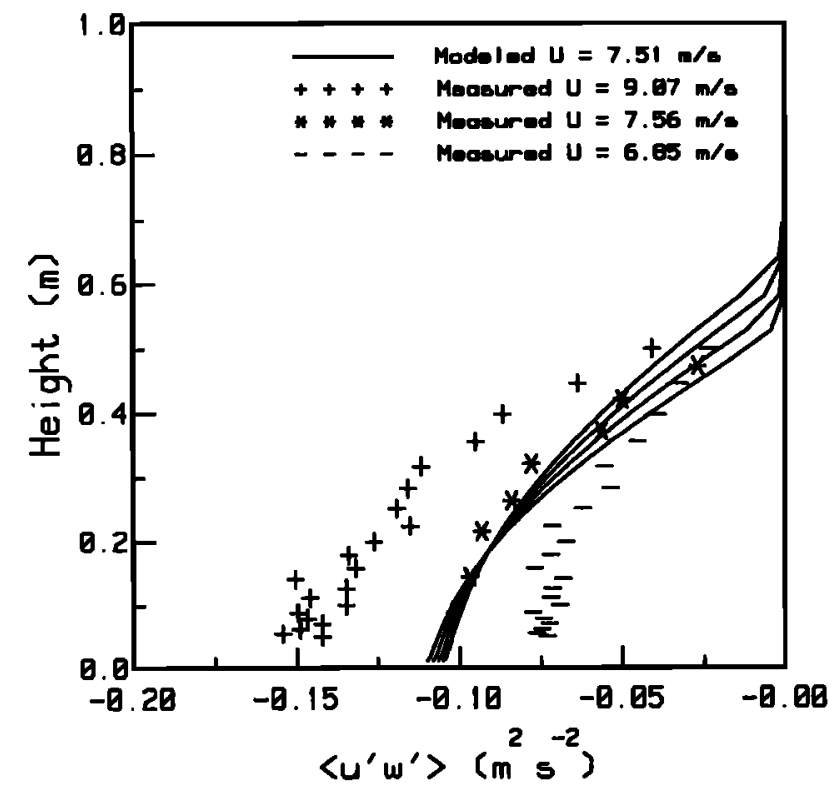

Figure 5. A comparison of the momentum flux measured at the indicated wind speeds at a fetch of $30 \mathrm{~m}$ in the IMST tunnel with the Eulerian model results. The group of curves depicts the growth of the simulated boundary layer through flux profiles determined at $27,29,31$, and $33 \mathrm{~m}$ (from right to left, looking at the top of the curves) for a wind speed of $7.5 \mathrm{~m} \mathrm{~s}^{-1}$.

by single profiles as used in part 1 (even without the droplets). Owing to the mixing of the air by the turboprop and heat exchangers, the air enters the test section with approximately constant values of specific humidity and temperature. As the air moves along the length of the tunnel, it interacts with the water surface through molecular and eddy exchange. This interaction is shown in Figures 3 and 4, which indicate good agreement between measured and modeled results at a fetch of $30 \mathrm{~m}$. These measurements were made using a thermocouple psychrometer system provided by the University of Washington using chromel constantan thermocouples. These devices are believed to be accurate to $\pm 0.2{ }^{\circ} \mathrm{C}$; however, cold spikes caused by droplets impacting the sensors are expected to increase the uncertainty in these measurements [Katsaros et al., 1994].

The result of primary interest for droplet dispersion is shown in Figure 5, which displays the $-\overline{u w}$ component of the Reynolds-stress tensor computed from

$$
-\overline{u w}=v_{T} \frac{\partial \bar{U}}{\partial z}
$$

with carefully conducted measurements made at a fetch of 30 m over a range of wind speeds by Giovanangeli and LeCalve [1990] at IMST. The group of curves representing the model results at several fetches shows that the model gives good agreement with the measurements made at the same wind speed at a fetch of $30 \mathrm{~m}$. These curves are representative of a developing LBL and indicate that the growing surface layer reaches a height of approximately $0.2 \mathrm{~m}$ at $30 \mathrm{~m}$. The implications of this developing surface layer on droplet dispersion is addressed in section 3 .

\section{Droplet Dispersion Modeling}

Several Eulerian approaches have been successfully applied to the study of turbulent diffusion of discrete particles in boundary layer flows. These include the studies of $\mathrm{Ling}$ and Kao [1976], Ling et al. [1978, 1980], Burk [1984], Mostafa and Mongia [1987], Stramska [1987], and Rouault et al. [1991], among others. However, the restraints placed on these models often restrict their application to either very small, highly concentrated particles or simple flow geometries with a homogeneous source of particles that can then be studied using budget equations.

Note that these constraints are not always a drawback. For example, in highly concentrated flows, where the particleparticle interactions are not negligible, it is generally much easier to include these effects in a Eulerian scheme than a Lagrangian one. Additionally, the use of budget equations can provide a means of studying the effects of particle interaction with the scalar fields through the use of source/sink relationships, which are difficult to include in a purely Lagrangian scheme. This approach has been successfully employed by Rouault et al. [1991], who studied the effect of droplet evaporation on the scalar fields of temperature and humidity.

The simulation of the movement of heavy particles involves parameterizations to account for the effects of gravity and inertia. These parameterizations generally require the tuning of adjustable constants through comparison with experimental data [e.g., Rouault et al., 1991]. Unfortunately, these calibration measurements are difficult to come by, several notable exceptions being the studies of Snyder and Lumley [1971] and the Humidity Exchange Over the Sea (HEXOS) in a Simulation Tunnel (HEXIST) experiments [e.g., Andreas et al., 1995]. There is also a question as to the universality of these constants when they are applied to more complicated flows. Finally, as in any Eulerian model, discrete sources of particles are difficult to include in the calculation domain and generally require subgrid-scale parameterizations that are often difficult to quantify.

Therefore we feel that the Lagrangian approach is the best alternative if one is concerned with the dispersion of lowconcentration, heavy particles from discrete or nonuniform sources (e.g., from spume droplets produced at the wave crests) and in fluid flows with complicated geometries (e.g., over waves or within a surf zone). The approach also allows for much more flexibility in specifying boundary conditions. For example, a Lagrangian scheme can easily include boundary conditions where the particles either rebound or stick, depending on the type of surface one is trying to simulate. It is also especially advantageous in situations where the physicochemical characteristics of the particles change rapidly when they experience a highly inhomogeneous environment. In this instance, time rate of change equations, which may depend upon the local conditions, as well as on the particle's history, are easily included in the model as long as these equations are known.

The Lagrangian model is described in detail in part 1. Briefly, the model relies on a finite difference form of the Langevin equation for the fluctuating components of the droplet's velocity due to turbulence. When the mean droplet 
fall velocity $\overline{W^{P}}$ is added to this fluctuating component $w^{P}(t)$, the instantaneous vertical velocity is given by

$$
\begin{gathered}
W^{p}(t+\Delta t)=\left(1-\frac{\Delta t}{\tau_{w}^{p}}\right) W^{P}(t) \\
+\sigma_{w}^{p}\left(\frac{2}{\tau_{w}^{p}}\right)^{1 / 2} \zeta(t)+\frac{\Delta t}{\tau_{w}^{p}} \overline{W^{p}}
\end{gathered}
$$

where $\tau_{w}^{p}$ is the droplet integral timescale, $\Delta t$ is the time step, $\sigma_{w}^{p}$ is the square root of the droplet vertical velocity variance, and $\zeta(t)$ is a random number drawn from a Gaussian distribution with zero mean and variance $\Delta t$. The expressions for $\tau_{w}^{p}$ and $\sigma_{w}^{p}$ are then derived from the equation of motion for small, heavy droplets given by

$$
\frac{D w^{p}}{D t}=-\frac{K}{\alpha} w^{R}=\frac{K}{\alpha}\left(w^{f}-w^{p}\right)
$$

where the superscripts $f$ and $R$ denote the fluid and relative velocities, respectively, and the total derivative denotes motion following the droplet. The parameter $K$ represents the ratio of the Stokes velocity to the mean relative fall velocity, and $\alpha$ is the response time for droplets that ideally obey Stokes law.

The approach given in part 1 yields the following expression for the vertical velocity variance:

$$
\sigma_{w}^{p^{2}}=\frac{\sigma_{w}^{2}}{(1+\chi)}
$$

where

$$
\chi=\frac{\alpha}{K \tau_{L}}
$$

The parameter $\chi$ is the ratio of the droplet response time to the Lagrangian integral time scale $\tau_{L}$. This parameter thereby determines how the droplet reacts to the turbulent motion of the surrounding fluid, e.g., as the droplet encounters smaller eddies as it nears the surface (smaller $\tau_{L}$ ), the influence of the turbulence on the droplet motion diminishes because the droplet can no longer react to these smaller eddies due to its inertia. The height at which this begins to occur is determined by the droplet's size as reflected by its response time $\alpha / K$. The equivalent situation occurs as the turbulence intensity increases (again, smaller $\tau_{L}$ ) such that its response time becomes too large to permit the droplet to react fast enough to all scales of the turbulence.

The integral timescale is a measure of the persistence of the droplet's velocity as it moves through the fluid. This coefficient is derived by determining the droplet autocorrelation coefficient in an approach similar to that used by Meek and Jones [1973]. The droplet integral timescale is then determined by integrating the autocorrelation function over time, which yields

$$
\tau_{w}^{p}=\frac{\tau_{L}}{\Lambda}(1+\chi)
$$

where

$$
\Lambda=\left[1+\left(\overline{W^{P}} / \sigma_{w}^{p}\right)^{2 / 2}\right.
$$

The parameter $1 / \Lambda$ accounts for the decorrelating effect of the droplet falling out of a fluid parcel where the fluctuating fluid velocities are highly correlated over small time steps. In past studies this parameter has been tuned to fit experimental data by multiplying ( $\left.W^{P} / \sigma_{w}^{p}\right)^{2}$ in (32) by a constant ranging between 0.3 and 1 [e.g., Mostafa and Mongia, 1987; Wells and Stock, 1983]. In this study we continue to use the formulation given in part 1; that is, the constant is left equal to 1 . We address the implication of this choice in the discussion given below.

The Lagrangian integral timescale is commonly defined in engineering literature [e.g., Mostafa and Mongia, 1987] using the output of the $k-\epsilon$ model as $\tau_{L}=\beta k / \epsilon$, where $\beta$ ranges between 0.15 and 0.6 . If we equate this expression with the expression used in part 1 , we obtain

$$
\tau_{L}=\frac{v_{T}}{\sigma_{w}^{2}}=0.18 \frac{k}{\epsilon}
$$

The vertical velocity variance is parameterized in terms of the kinetic energy by combining (21) with the results from Mestayer [1980] to obtain

$$
\sigma_{w}^{2}=1.69 C_{\mu}^{1 / 2} k
$$

\section{Gwaihir}

With these parameters we have all the necessary expressions to simulate the turbulent dispersion of heavy particles once the Eulerian fields are available from the $k-\epsilon$ code. Therefore, after a steady state solution of a particular flow configuration is found, the velocity and scalar values are passed to the Lagrangian section of the code in order to determine the parameters in the above expressions. These parameterizations are then used with (27) to compute the trajectories of evaporating droplets. The amount of trajectories begun over a specified time $\mathrm{T}$ is determined by a user-defined source function. As these trajectories are computed, the Lagrangian code keeps track of the droplet concentration $q_{D}$, sensible heating rate $S_{H}$, and water vapor production $S_{q}$ in each cell. Once the Lagrangian portion of the code has ejected all of the droplets determined by the source function over the time $T$, the Eulerian portion of the code is then rerun with the new nonzero values of $q_{D}, S_{q}$, and $S_{H}$. This process is repeated until the $k-\epsilon$ code, upon completion of the last Lagrangian run, reaches a steady state over an interval less than $T$.

The source of droplets is determined from the surface source function, which gives the number of droplets per unit time, per unit area produced at the surface. Since the droplet measuring devices were situated slightly downwind of the 30-m-long bubbler array used during the CLUSE campaign [Mestayer et al., 1990], the Gwaihir source function was determined by matching the model output with droplet measurements made at a height of $0.2 \mathrm{~m}, 0.5 \mathrm{~m}$ downwind of the simulated source. 
The total number of droplets over a radius interval between $r-\Delta r / 2$ and $r+\Delta r / 2$ ejected from a prescribed area (where $\Delta r$ is the radius increment) is given by

$$
N(r)=N_{T}(r) \mathrm{T} \Delta r \Delta x \Delta y
$$

where $N_{T}(r)$ is the surface source function, Tis the total time that the source is on, $\Delta x$ is the length of the area, and $\Delta y$ is its width (set equal to unity in this 2-D simulation). Since Gwaihir uses a horizontal grid spacing of $\Delta x=0.5 \mathrm{~m}$, the source is divided up into 60 sections.

The droplets produced in each section are then released at their ejection height. This height corresponds to the height to which a jet droplet is ejected by a bubble bursting. The height and number of droplets ejected are a function of bubble size, and we have used the data given by Blanchard and Woodcock [1957] to determine these parameters as described in part 1.

As the released droplet moves through the model domain, the values needed in the Lagrangian model parameterizations are determined from a linear average of the four nearest Eulerian grid points in order to find $U(X, Z), W(X, Z)$, and $\Phi(X, Z)$ at $X, Z$. Owing to the staggered grid, this involves keeping track of four different indices corresponding to $x, x x, z$, and $z z$. If the droplet falls below $z z_{1}$, the values of the variables are found using the same wall functions employed in the Eulerian model. If the droplet falls below $z_{o}$, it is assumed to stick to the surface, in which case another trajectory is begun. A new droplet trajectory is also begun if a droplet is carried out of the calculation domain.

The concentration of droplets over a specified size range is calculated by accumulating the time these droplets spend in each cell. The accumulated time, divided by the total time that the source is on, divided by the volume of the cell is a measure of the droplet concentration. The droplet volume concentration (droplet volume, per unit volume, per radius increment) in each cell is then computed from

$$
\frac{d V(r)}{d r}=\frac{4}{3} \frac{\pi r^{3}}{V \mathrm{~T} \Delta r} \sum \Delta t
$$

where the summation is for each cell over the advection time $T$, and $V=\Delta x \Delta y \Delta z$ is the volume of each cell.

The source functions of $\rho_{a} c_{p} \Theta$ and $Q$ are the means by which the evaporating droplets interact with the fields of sensible heat and moisture. These functions can act as either a source or sink of sensible heat and moisture depending on the ambient conditions. Therefore $Q$ in (6) is actually the total specific humidity

$$
Q(x, z)=q_{\nu}(x, z)+q_{L}(x, z)
$$

where $q_{v}$ is the specific humidity due to water vapor and $q_{L}$ is the contribution due to liquid water other than the spray droplets (e.g., fog). Here we are assuming that the fog droplets are small enough that they can be properly modeled by (6); that is, the fog droplets are small enough to be treated as passive scalars. On the other hand, the spray droplets are too large for such treatment and are instead modeled separately by the Lagrangian code.
In breaking down the specific humidity in this fashion the source/sink functions become solely a function of droplet evaporation/condensation. The interaction between the evaporating droplets and the scalar fields is then simulated by releasing all the droplets produced during a given period of time and accumulating the sensible heat and moisture they consume or release in each cell. The functions used in this model are given as

$$
\begin{gathered}
S_{H}=-\frac{4 \pi}{V \mathrm{~T}} \sum r k_{a} f_{H}\left(T_{a}-T_{s}\right) \Delta t \\
S_{q}=-\frac{4 \pi \rho_{\rho}}{V \mathrm{~T} \rho_{a}} \sum r^{2} \frac{d r}{d t} \Delta t
\end{gathered}
$$

where the summation is over all droplets in each cell, $p_{p}$ is the density of the droplet, $f_{H}$ is the ventilation coefficient for heat diffusion [Beard and Pruppacher, 1971], $k_{a}$ is the thermal conductivity of air, and $T_{a}$ and $T_{s}$ are the air and droplet surface temperatures, respectively.

The evaporation rate, $d r / d t$, is determined using the function given by Beard and Pruppacher [1971] using modification proposed by Andreas [1989] for curvature effects, while the droplet surface temperature is governed by the functions described in part 1 . The droplet surface temperature is assigned the value of the water surface at the time of its release. Initialized in this way, Gwaihir explicitly models the initial transfer of sensible heat from droplet to air when the sea surface temperature exceeds the air temperature (unstable conditions) as described by Andreas et al. [1995], as well as the eventual cooling (heating) of the atmosphere due to evaporation (condensation).

The virtual temperature required in (4), (14), and (15) is then determined by considering all three sources of moisture [Stull, 1988]

$$
\begin{gathered}
\Theta_{v}(x, z)= \\
\Theta(x, z)\left[1+0.61 q_{v}(x, z)-q_{L}(x, z)-q_{D}(x, z)\right]^{(40)}
\end{gathered}
$$

where $q_{D}$ is the contribution to the total specific humidity from the droplets; that is, it is found by integrating the droplet volume concentration in each cell. In practice, $q_{L}$ is equal to zero unless $Q$ is greater than its saturation value. If supersaturation occurs, then $q_{\nu}(x, z)$ is equal to $q_{\text {sat }}(x, z)$ and $q_{L}(x, z)$ is equal to $Q(x, z)-q_{\mathrm{sat}}(x, z)$.

These additional sources of moisture are included in the buoyancy flux using the approach given by Stull [1988]

$$
\begin{gathered}
\overline{w \theta_{v}}=\overline{w \theta}\left[1+0.61 q_{v}-q_{L}-q_{D}\right] \\
+\Theta\left[0.61 \overline{w q}-\overline{w q_{L}}-\overline{w q_{D}}\right]
\end{gathered}
$$

where we are ignoring the triple products found in its full derivation. The fluxes are defined by breaking down the moisture terms as described above and using 


$$
-\overline{w C^{\prime}}=\operatorname{Pr}_{T} \mathrm{v}_{T} \frac{\partial C}{\partial z}
$$

where $C=q_{\nu}, q_{L}, q_{D}$ and we assume, following Rouault et al. [1991], that the turbulent Prandtl and droplet Schmidt numbers are equal.

\section{Gwaihir Results}

In this section we concentrate on how we have addressed some of the earlier model's deficiencies by combining it with the Eulerian code. Specifically, we examine the improvement between the model and measurement comparisons due to a more realistic simulation of the turbulent field through which the droplets are dispersing. Second, we demonstrate the improvements in our simulations due to the ability of Gwaihir to permit interaction between the droplets and the scalar fields. Finally, we examine the extent to which the droplets modify the scalar fields and examine how this effect modifies the fluxes of sensible and latent heat.

\subsection{Influence of the Turbulent Flow Field on Droplet Dispersion}

In section 2.5 we demonstrated that the $k-\epsilon$ model is capable of accurately simulating a 2-D developing surface layer. We now examine how the improved simulation of the turbulent flow field affects the output of the Lagrangian component. In Figure 6, we begin our comparison between Gwaihir and the droplet measurements taken during the CLUSE campaign. It shows excellent agreement between Gwaihir (at a fetch corresponding to $30.5 \mathrm{~m}$ ) and measurements for all sizes and

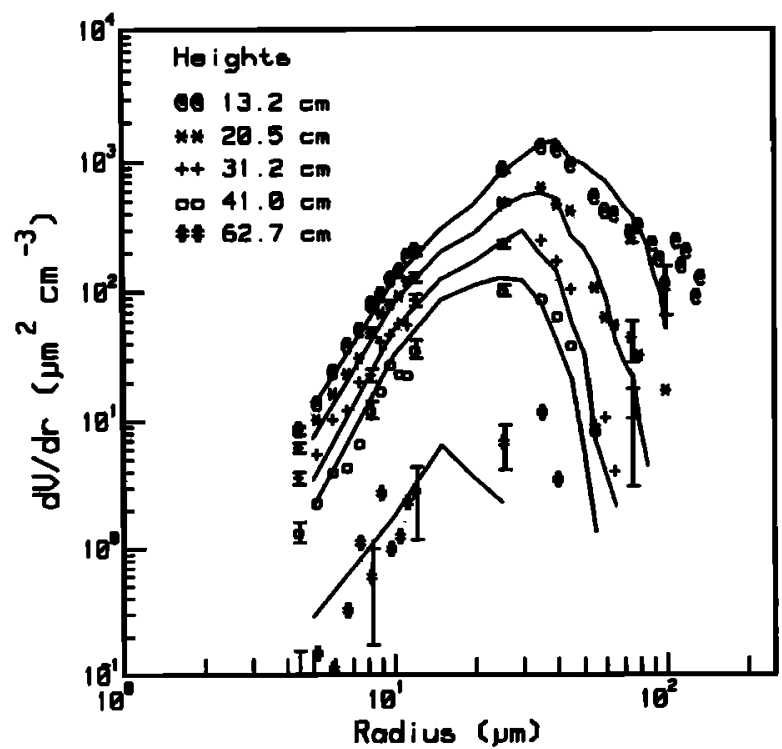

Figure 6. Droplet volume spectra as a function of droplet radius. The solid lines represent the model results, while the symbols represent data measured at the indicated heights. The error bars are computed at every fifth data point from $\mathrm{dV} / d \mathrm{r} \mathrm{x}$ $(1 \pm 1 / \sqrt{ } N$, where $N$ is the number of droplets counted over the size interval. The conditions during the run were a relative humidity of $95 \%$ and a nominal wind speed of $7.5 \mathrm{~m} \mathrm{~s}^{-1}$.

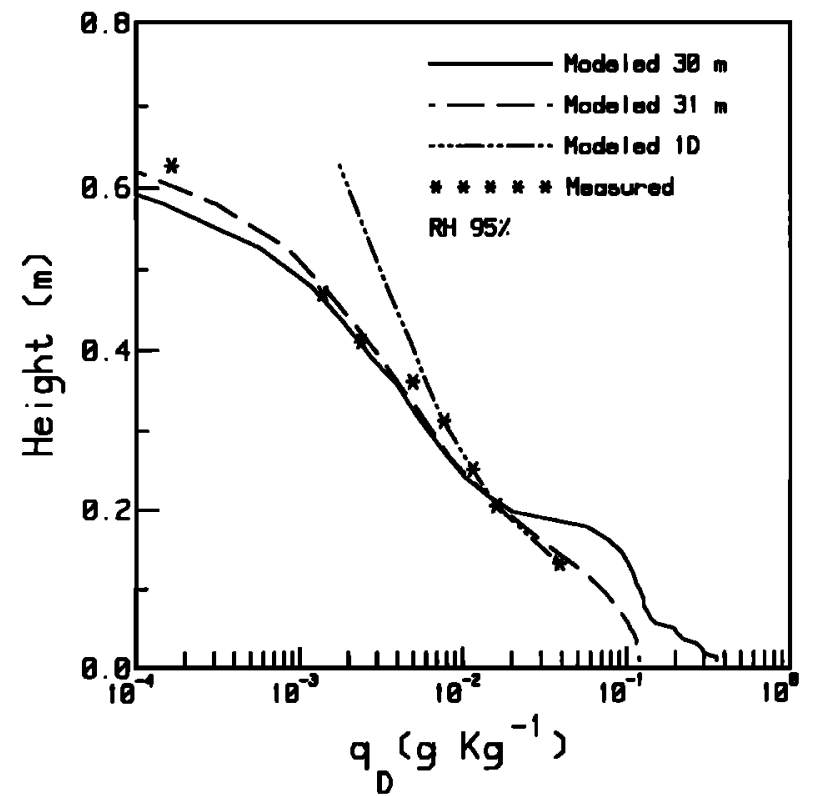

Figure 7. Profiles of total liquid water content $q_{D}$, calculated by integrating over the measured and modeled droplet spectra at each height. The symbols represent data measured at the indicated heights. The solid line represents Gwaihir results taken directly over the simulated source of droplets, while the dashed line shows the profile $1 \mathrm{~m}$ beyond the end of this source. The dash-dotted line reproduces the one-dimensional model results given by Edson and Fairall [1994]. The conditions during the run were a relative humidity of $95 \%$ and a nominal wind speed of $7.5 \mathrm{~m} \mathrm{~s}^{-1}$.

heights, except for the largest droplets at $63 \mathrm{~cm}$. This is perhaps a result of the merging of two boundary layers in the actual tunnel, which we have not tried to model in this simulation.

Upon close inspection of Figure 6 we see that Gwaihir is slightly underestimating the vertical dispersion of the larger droplets. The agreement between the model and measurements at the largest sizes could be improved by including a fractional constant in (32) as described above. However, its inclusion would worsen the agreement at smaller sizes. A more likely explanation for the disagreement is that we are simply pushing Gwaihir too far; that is, we are using a 1-D Lagrangian model in a 2-D flow. A better alternative is to make the Lagrangian portion of the code 2-D by including a correlated horizontal component of the droplet velocity as did Ley and Thomson [1983]. Additionally, the assumptions that lead to its development suggests that it works best in homogeneous turbulence, which is clearly not the case in the LBL. In fact, we expect Gwaihir to work better in simulations of a marine surface layer, where these conditions are better realized.

Even with these minor shortcomings, Figures 7 and 8 indicate that we have improved the model simulations (compared with the results given in part 1) by simply impro-ving the simulation of the turbulent flow field, rather than trying to tune the Lagrangian model parameters. This is evident in the profiles of $q_{D}$ given in Figure 7, which is computed by approximating 


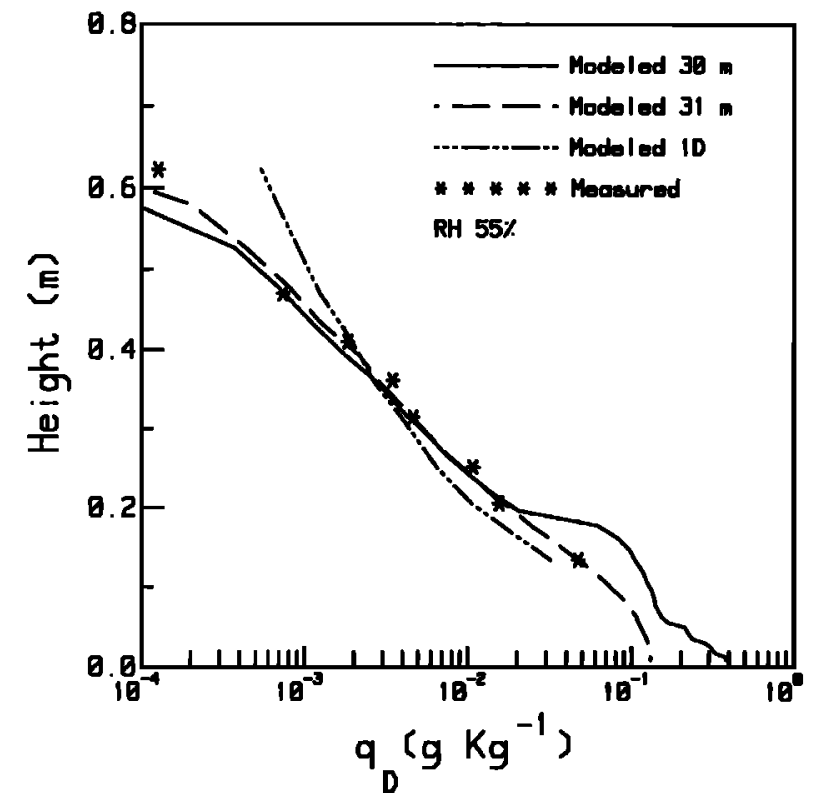

Figure 8. Profiles of total liquid water content $q_{D}$, for a relative humidity of $55 \%$ and a nominal wind speed of $7.5 \mathrm{~m} \mathrm{~s}^{-1}$. The lines are as denoted in Figure 7.

the area under the curves shown in Figures 6 and 9 in part 1 using

$$
q_{D}=\frac{\rho_{p}}{\rho_{a}} \sum_{i=1}^{N}\left(\frac{d V}{d r}\right)_{i} \Delta r_{i}
$$

where $N$ is the total number of radius increment bins and the density units are chosen to give grams per kilogram. Figure 7 shows that either version of the model gives excellent agreement with the measurements within the constant flux layer. Recall that the constant flux is assumed to exist throughout the $1-\mathrm{D}$ boundary layer simulated in part 1 . This is the reason why Gwaihir gives much better agreement as we near the top of the boundary layer, where the momentum flux tends toward zero, which causes a drastic reduction in the vertical dispersion of the droplets.

Figure 8 shows that Gwaihir does a better job of handling droplet evaporation than the 1-D model, which tended to overevaporate the droplets. This is due to the inclusion of droplet feedback mechanisms (i.e., the moistening of the near surface which lessens the amount of evaporation), as well as the general improvement of the mean profile simulations of temperature and moisture. In fact, this latter effect is most likely the cause for the improvement, for reasons given in section 5.2.

\subsection{Influence of Droplets on the Scalar Fields}

The first step in the simulation is to compute the turbulent fields of velocity, temperature, and humidity in the absence of droplets. Therefore the $k-\epsilon$ model gives us an easy way to determine the effect of droplet evaporation on the scalar fields by examining the difference in the temperature and humidity profiles modeled with and without droplets. In Figure 9 we

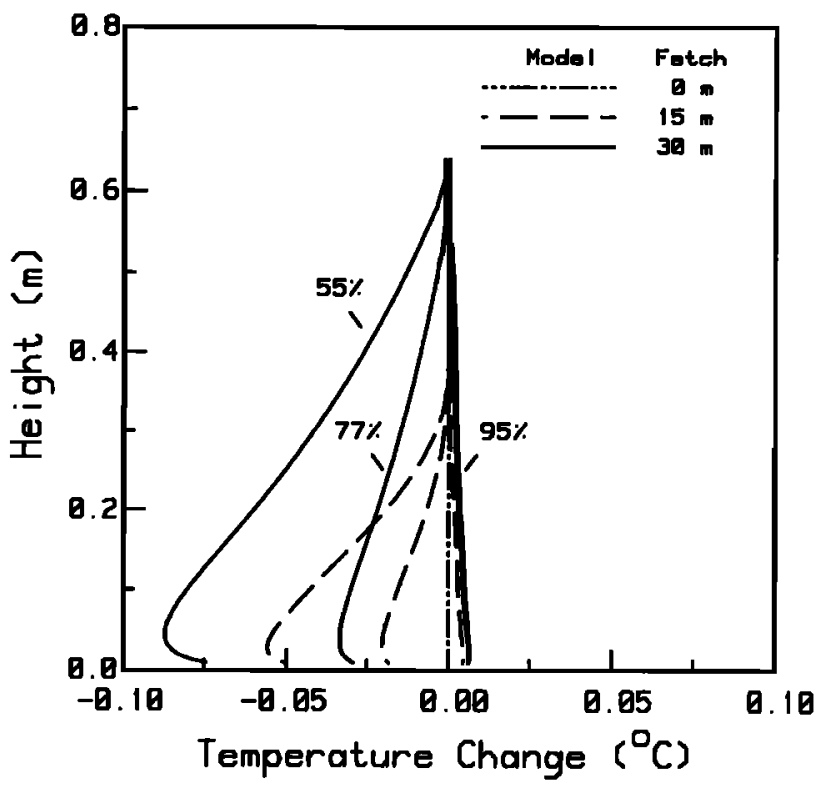

Figure 9. Profiles of the change in the model-derived temperature profiles due to the presence of droplets. The nominal wind speed was $7.5 \mathrm{~m} \mathrm{~s}^{-1}$ for all runs, while the relative humidity is as indicated on the profiles measured at $30 \mathrm{~m}(55 \%, 77 \%$, and 95\%).

depict the difference in temperature profiles due to the presence of droplets. The high humidity run in Figure 9 illustrates a case where the release of sensible heat from the droplets causes the near-surface air temperature to actually increase. This can be attributed to the large, positive water-air temperature difference and little evaporative cooling at high humidity.

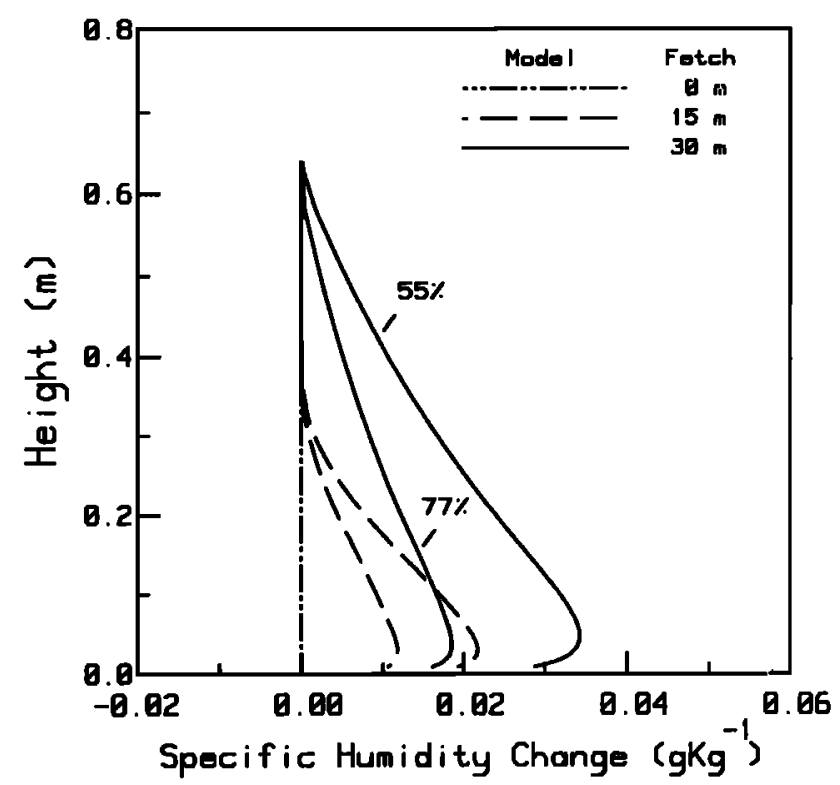

Figure 10. Profiles of the change in the model-derived specific humidity profiles due to the presence of droplets. The nominal wind speed was $7.5 \mathrm{~m} \mathrm{~s}^{-1}$ for all runs, while the relative humidity is as indicated on the profiles measured at 30 $\mathrm{m}(55 \%$ and $77 \%)$. 
The lower-humidity runs shown in Figures 9 and 10 give the expected result of lower near-surface temperatures and higher specific humidities in the presence of droplets due to their evaporation. The magnitude of this change is similar to the results of Rouault et al. [1991], with a maximum of approximately $0.1^{\circ} \mathrm{C}$ in temperature and $0.05 \mathrm{~g} \mathrm{k} \mathrm{g}^{-1}$ in specific humidity. This is a barely measurable change (in fact, the precision of the sensors may not be able to resolve it) and is smaller than the measurements shown in Figure 16 in part 1. As stated in part 1, we believe that this is a consequence of droplets wetting the "dry-bulb" thermometer resulting in an underestimation of the dry-bulb temperature and overestimation of the specific humidity. For this reason we believe that the improvement in the model's performance is most likely due to the inclusion of the 2-D simulation of the mean temperature and humidity profiles, rather than the droplet feedback mechanism.

\subsection{Influence of the Droplets on the Surface Energy Budget}

On the basis of the results given in section, 5.2, one might conclude that we should not expect droplets to have an appreciable effect on the surface energy budget. However, there are a number of reasons why this may not be the case for the marine boundary layer (MBL). For example, we have already shown that the intensity of $\overline{u w}$ in the LBL falls off rapidly with height. This decrease has a particularly adverse effect on large droplet lispersion as evidenced by Figures 7 and 8 , where the majority of the potential moisture from the droplets is found below the highest height of $0.18 \mathrm{~m}$, indicating that the largest droplets (which dominate the total water content) are rarely found above this level. This effect is also responsible for the reduction of the droplet concentration profile, as one moves 0.5 $\mathrm{m}$ downwind of their source, due to the rapid fallout of the largest droplets.

In fact, direct measurements of droplet profiles made from a wave follower by De Leeuw $[1986,1987]$ have shown that the gradient over the ocean is much smaller than that measured in the laboratory. While the mechanisms responsible for the enhanced mixing remain a hotly debated topic, it has been postulated that the reduced gradient may be the result of waveinduced motions and/or spume droplet production (e.g., see the discussion by $W u[1990]$ and De Leeuw [1990]). These spume drops are generated when spray is directly tom off the wave crests in high-wind conditions and are addressed in section 6 .

Finally, in the CLUSE setup a droplet ejected at the tunnel entrance and kept aloft for the entire length of the test section $(30 \mathrm{~m})$ has approximately $4 \mathrm{~s}$ (at a nominal wind speed of 7.5 $\mathrm{m} \mathrm{s}^{-1}$ ) to interact with the turbulent fields of temperature and humidity. The work of Andreas [1990] has shown that droplets having an initial radius smaller than $20 \mu \mathrm{m}$ are able to exchange $68 \%$ of their mass with the environment in $4 \mathrm{~s}$. We can see the result of this exchange in Figure 10, where the contribution of the droplets to the moisture field increases significantly with fetch. However, this also means that much less than half of the droplet volume (found by integrating under the curves in Figure 6) has a chance of contributing to the moisture field.

\section{Marine Boundary Layer Simulation}

The above discussion is particularly relevant to open ocean research because we have evidence that the source of droplets generated at IMST is representative of high-wind conditions over the open ocean. This is based on the comparison between the CLUSE source function and the estimates of the over-ocean function given by Andreas [1992] shown in Figure 11, which attempts to include the additional source of droplets arising from spume drop production. Figure 11 shows that the two functions are comparable for wind speeds between 15 and 18 $\mathrm{m} \mathrm{s}^{-1}$. The review by Andreas et al. [1995] placed this function on the high end of the most probable estimates of spray droplet production. Therefore this function is expected to maximize the influence of spray droplets on the near-surface energy budget, although even this assumption is far from certain due to our lack of knowledge concerning the generation of the largest (spume) drops.

In order to examine the influence of the droplets under highwind conditions in the MBL, we conducted two separate simulations of a fully developed atmospheric surface layer using the Andreas [1992] source function. In both simulations we initialize Gwaihir with a constant flux layer using a $10-\mathrm{m}$ wind speed $U_{10}$ of $18 \mathrm{~m} \mathrm{~s}^{-1}$, an air temperature of $20^{\circ} \mathrm{C}$, a water temperature of $22^{\circ} \mathrm{C}$, and a relative humidity of $80 \%$. Periodic boundary conditions are used to allow the droplets to interact with the turbulent fields as long as they are airborne. The velocity is fixed at the upper boundary, while the temperature and humidity values are allowed to adjust so that the flux remains constant across the upper boundary. These conditions are specifically chosen for comparison with the analytical model results given by Andreas et al. [1995].

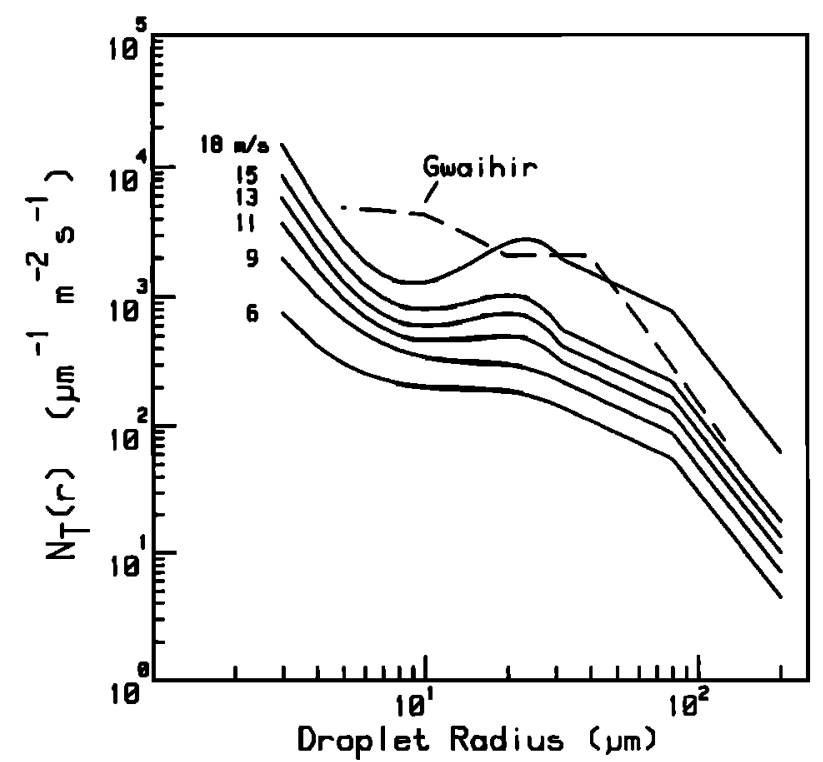

Figure 11. A comparison of the Gwaihir source function with the parameterization of the over ocean source function given by Andreas [1992]. 


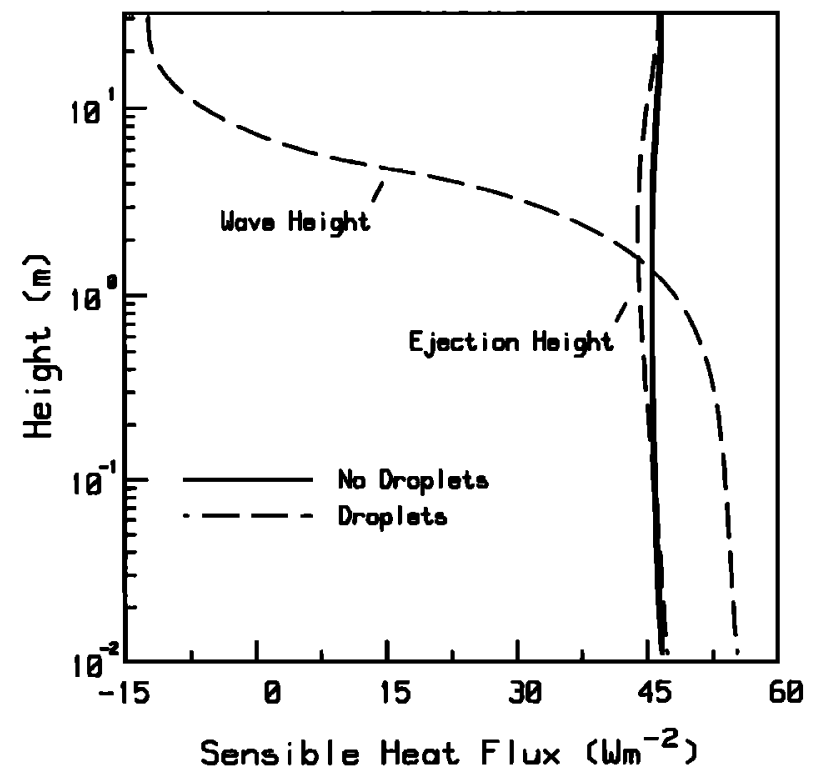

Figure 12. The model-derived sensible heat fluxes computed in the absence and presence of droplets released at two difference locations. The conditions during the run were a wind speed of $18 \mathrm{~m} \mathrm{~s}^{-1}$ and air temperature of $20^{\circ} \mathrm{C}$ at $10 \mathrm{~m}$, a water temperature of $22^{\circ} \mathrm{C}$, and a relative humidity of $80 \%$.

The current version of Gwaihir does not attempt to directly model the influence of waves. However, we believe we can place some quantitative limits on the role they play in droplet dispersion and evaporative processes through the height at which we release the droplets. In the first simulation we release the droplets at their ejection height, while in the second simulation we release the droplets at the significant wave height determined by

$$
A_{1 / 3}=0.015 U_{10}^{2}
$$

The latter is intended to simulate a situation where the spume droplets are sheared off the wave crests and immediately find themselves at a considerable distance from the mean surface. Andreas [1992] has argued that $A_{1 / 3}\left(\overline{W^{P}}\right)^{-1}$ is an appropriate timescale in his model, both because of the spume droplet effect and because it serves as a means to parameterize the increase of turbulence intensity with wind speed. However, because turbulent dispersion is already included in our model, we expect this simulation to give a best case scenario for droplet dispersion. This simulation should therefore approximate the upper bound on the possible droplet influence under these conditions. The release of the droplets from their ejection height is far less favorable for droplet dispersion and is expected to place a lower bound on the interactive processes for this particular source function.

The droplet's influence on the latent and sensible heat fluxes for the two runs are shown in Figures 12 and 13, where the fluxes are calculated from (9) and (10). The change to the mean humidity and temperature profiles are also shown in Figures 14 and 15 for comparison with the LBL results.

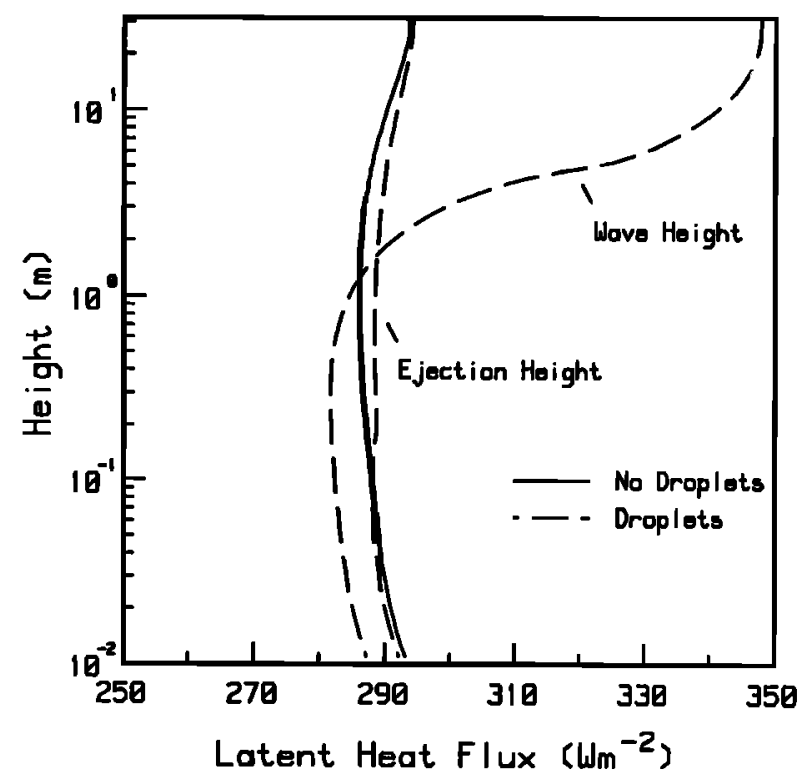

Figure 13. The model-derived latent heat fluxes computed in the absence and presence of droplets released at two difference locations. The conditions during the run are as in Figure 12.

Figures 12 through 15 show that the influence of droplet evaporation on the mean profiles and surface heat fluxes is strongly affected by the height at which the droplets are released.

The droplets released at their ejection height have a minimal impact on the latent and sensible heat fluxes and produce changes to the mean profiles that differ only slightly from the laboratory results. However, the droplets released at the wave

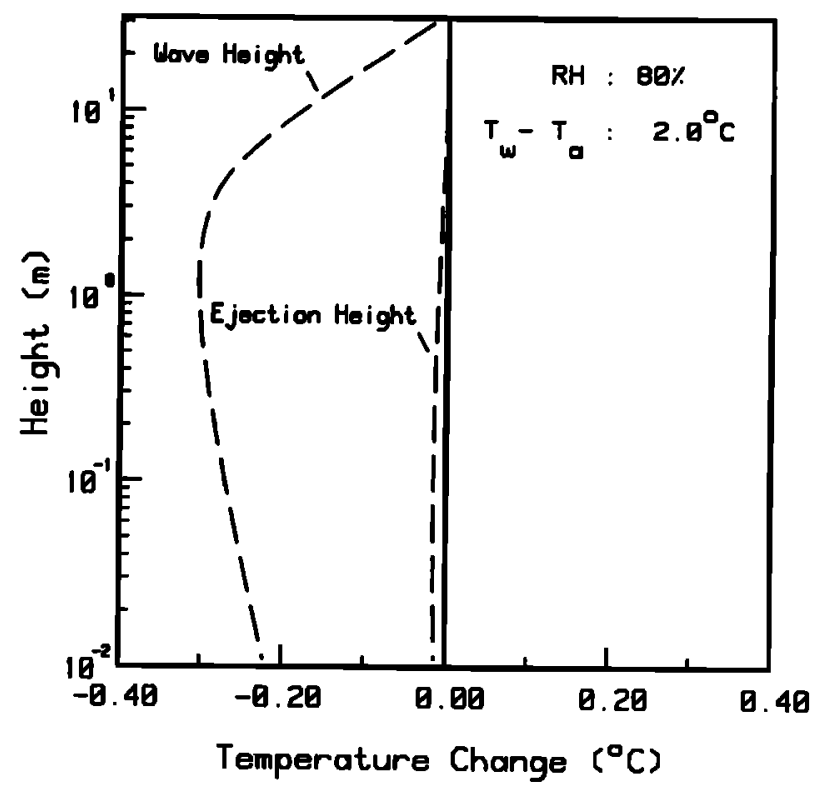

Figure 14. Profiles of the change in the model-derived temperature profiles due to the presence of droplets in our marine boundary layer (MBL) simulation. The conditions during the run were a relative humidity of $80 \%$ and a $10-\mathrm{m}$ wind speed of $18 \mathrm{~m} \mathrm{~s}^{-1}$. 


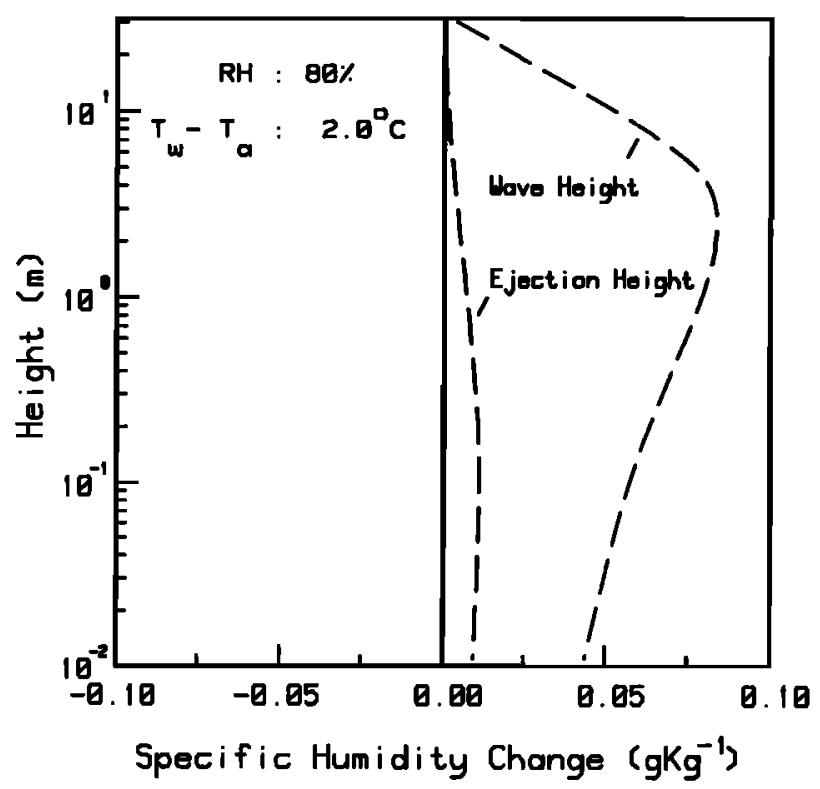

Figure 15. Profiles of the change in the model-derived specific humidity profiles due to the presence of droplets in our MBL simulation. The conditions during the run were a relative humidity of $80 \%$ and a $10-\mathrm{m}$ wind speed of $18 \mathrm{~m} \mathrm{~s}^{-1}$.

height (4.86 $\mathrm{m}$ in this simulation) significantly increase an already substantial latent heat flux by $20 \%$ at the top of model domain. The relatively weaker sensible heat flux is decreased by more than $100 \%$. These finding agree very well with the results of Andreas et al. [1995], which found that the total droplet generated heat flux was roughly $30 \%$ of the interfacial (bulk) flux.

An interesting feature of these profiles is the apparent asymmetry of the droplet feedback effects described in part 1 and by Andreas et al. [1995]. These effects act to reduce the latent heat flux near the surface and increase it above. Fairall et al. [1995] hypothesized that this feedback could be included in the model of Andreas [1992] by assuming that only a fraction of the total droplet generated heat fluxes appears above the droplet evaporation layer. The asymmetry shown in Figures 12 and 13 suggests that this fraction is greater than the $50 \%$ used by Fairall et al. [1995].

\section{Conclusions}

In this paper we have shown that the Lagrangian model simulations given in part 1 have been even further improved by simply improving the simulation of the turbulent fields through which the droplets are dispersed. In the field, where it is difficult to measure even mean profiles near the surface, we can use the $k-\epsilon$ model to provide the required Lagrangian model parameters using measurements from the surface and at some reference height to initialize the Eulerian model. The droplet model can then be used to examine the influence of the droplet evaporation and sensible heat release on the surface energy budget using a variety of wind-dependent source functions once reliable field-based source function estimates are available.
Alternatively, Gwaihir could be used with the few nearsurface profile measurements that are available to estimate the source function. This would be accomplished by adjusting the model source function until the modeled profiles match the measurements. The major problem with this approach is our lack of knowledge of how spume drops are produced over the ocean. This leads to a great deal of uncertainty in how to parameterize their production in numerical models. However, current research efforts focusing on spume drop production should improve these parameterizations in the near future.

Our results indicate that an increase in turbulence intensity due to high winds does not significantly increase the effect that evaporating jet droplets have on the scalar fields of temperature and humidity. The principal reason for this is that the turbulence is still not strong enough at the droplet's ejection height to overcome the fall velocity of the largest droplets. However, the potential for substantial modification of the surface heat fluxes exists if the presence of waves acts to eject the droplets higher, permitting the larger droplets to remain aloft for longer periods of time. Therefore we need to gain a better understanding of how the droplets are generated at high wind speeds (i.e., as spume and/or jet drops), as well as how the waves affect the dispersion of these droplets once airborne.

An initial attempt to study the waves effect has been reported by Andreas et al. [1995] using modifications to the model described in part 1. Although the relevance of these results as limited by the simplified flow field used to model the waveinduced velocity perturbations, these results clearly indicated that the presence of waves significantly increases the amount of vertical dispersion. Therefore the logical next step in the development of Gwaihir is to adapt it more fully to over-ocean conditions by introducing a wavy lower surface and using the $k-\epsilon$ model to simulate the flow field. We expect to produce a more accurate simulation of the flow over waves by using an approach similar to the modeling work of Gent and Taylor [1976].

Gwaihir will also have to determine a way to simulate the release of spume droplets if we wish to accurately model the effect of spray droplets under high-wind conditions. This might involve testing proposed source functions of spume droplets and releasing these droplets only at the wave crests. Of course, the inclusion of these droplets leads to a number of yet unresolved questions about their generation, such as the appearance of flow separation over breaking waves. However, we will leave those topics for future discussion.

Finally, the effects introduced by modeling droplets composed of seawater, rather than fresh water, would have to be included. This would primarily involve changes to the equations governing evaporation rate and determination of how to deal with the residual sea-salt droplet that remains behind after evaporation. Such droplets could have long residence times and are of importance in processes involving cloud physics and atmospheric optics.

Acknowledgments. Support for this research was provided by the National Science Foundation under grants INT-8907676 and OCE9115227 and by the Centre National de la Researche Scien-tifique. J. 
Edson acknowledges support from the Office of Naval Research under grant N00014-95-1-0021 during the final preparation and publication of this article. This is Woods Hole Oceanographic Institution contribution 8794 and HEXOS contribution 47.

\section{References}

Andreas, E. L., Thermal and size evolution of sea spray droplets, CRELL Rep. 89-11, 37 pp., U.S. Army Cold Reg. Res. and Eng. Lab., Hanover, N.H., 1989.

Andreas, E. L., Time constants for the evolution of sea spray droplets, Tellus, Ser. B., 42B, 481-497, 1990.

Andreas, E. L., Sea spray and the turbulent air-sea fluxes, $J$. Geophys. Res., 97, 11,429-11,441, 1992.

Andreas, E. L., J. B. Edson, E. C. Monahan, M. P. Rouault, and S. D. Smith, The spray contribution to net evaporation from the sea: A review of recent progress, Boundary Layer Meteorol., 72, 3-52, 1995.

Avva, R. K. S. J. Kline, and J. H. Ferziger, Computation of the turbulent flow over a backward-facing step using the zonal modeling approach, Rep.TF-33, Stanford Univ., Stanford, Calif., 1988.

Beard, K. V., and H. R. Pruppacher, A wind tunnel investigation of the rate of evaporation of small water drops falling at terminal velocity in air, J. Atmos. Sci, 28, 1455-1464, 1971.

Blanchard, D. C., and A. H. Woodcock, Bubble formation and modification in the sea and its meteorological significance, Tellus, 9, 145-158, 1957.

Burk, S. D., The generation, turbulent transfer and deposition of the sea-salt aerosol, J. Atmos. Sci., 41, 3040-3051, 1984.

Charnok, H., Wind stress on a water surface, Quart. J. Roy. Meteor. Soc., 81, 639, 1955.

Chorin, A. J., A numerical method for solving incompressible viscous flow problems, J. Comput. Phys., 2, 12-26, 1967.

De Leeuw, G., Vertical profiles of giant particles close to the sea surface, Tellus, Ser. B., 38B, 51-61, 1986.

De Leeuw, G., Near-surface particle size distribution profiles over the North Sea, J. Geophys. Res., 92, 14,631-14,635, 1987.

De Leeuw, G., Comment on "Vertical distributions of spray droplets near the sea surface: Influence of jet drop ejection and surface tearing" by J. Wu, J. Geophys. Res., 95, 9779 $9782,1990$.

Duynkerke, P. G., Application of the E- $\epsilon$ turbulence closure model to the neutral and stable atmospheric boundary layer, J. Atmos. Sci, 45, 865-880, 1988.

Edson, J. B., and C. W. Fairall, Spray droplet modeling, 1, Lagrangian model simulation of the turbulent transport of evaporating droplets, J. Geophys. Res., 99, 25,295-25,311, 1994.

Fairall, C. W., J. D. Kepert, and G. J. Holland, The effect of sea spray on the surface energy transports over the Ocean, Atmos.-Ocean System, in press, 1996.

Garratt, J. R., Review of drag coefficients over oceans and continents, Mon. Weather Rev., 105, 915-917, 1977.
Gent, P. R., and P. A. Taylor, A numerical model of the airflow above water waves, J. Fluid Mech., 77, 105-128, 1976.

Giovanangeli, J.-P., and O. Le Calve, The structure of the atmospheric turbulent flow in the close vicinity of wind waves, in Modelling the Fate and Influence of Marine Spray, edited by P. G. Mestayer, E. C. Monahan, and P. A. Beetham, Whitecap Rep. 7, pp. 49-64, Mar. Sci. Inst., Univ. of Conn., Groton, 1990.

Hirt, C. W., and F. H. Harlow, A general corrective procedure for the numerical solution of initial value problems, $J$. Comput. Phys., 2, 114-119, 1967.

Högström, U., Non-dimensional wind and temperature profiles in the atmospheric surface layer: A re-evaluation, Boundary Layer Meteorol., 42, 55-78, 1988.

Katsaros, K. B., J. DeCosmo, R. J. Anderson, S. D. Smith, C. Kraan, W. A. Oost, L. Hasse, K. Bumke, P. G. Mestayer, and M. H. Smith, Measurements of humidity and temperature in the marine environment during the HEXOS main experiment, J. Atmos. Ocean. Tech., 11(4), 964-981, 1994.

Lakehal, D., P. G. Mestayer, J. B. Edson, S. Anquetin, and J.-F.Sini, Eulero-Lagrangian simulation of atmospheric hydrometeor trajectories and impacts inside the urban canopy, Atmos. Environ., in press, 1996.

Landahl, M. T., and E. Mollo-Christensen, Turbulence and Random Processes in Fluid Mechanics, 154 pp., Cambridge Univ. Press, New York, 1986.

Launder, B. E., and D. B. Spalding, The numerical computation of turbulent flows, Comput. Methods Appl. Mech. Eng., 3, 269-289, 1974.

Lévi Alvarès, S., C. X. Zhang, and C. Sacré, Tests for a flow simulation around an isolated block: $k$ - $\epsilon$ model improvements, paper presented at 9th Symposium on Turbulence and Diffusion, Am. Meteorol. Soc., Roskilde, Denmark, 1990.

Lévi Alvarès, S., Three-dimensional numerical simulation of turbulent flow around buildings using the $k-\epsilon$ model, Doctorate thesis, Lab. de Mec. des Fluides, Ecole Cent. de Nantes, France, 1992.

Lévi Alvarès, S., and J. F. Sini, Simulation of diffusion within an urban street canyon, J. Wind Eng., 82, 114-119, 1992.

Ley, A. J., and D. J. Thomson, A random walk model of the dispersion in the diabatic surface layer, Q.J.R. Meteorol. Soc, 109, 848-880, 1983.

Ling, S. C., and T. W. Kao, Parameterization of the moisture and heat transfer process over the ocean under whitecap sea states, J. Phys. Oceanogr., 6, 306-315, 1976.

Ling, S. C., A. Saad, and T. W. Kao, Mechanics of multiphase fluxes over the ocean, in Turbulent Fluxes through the Sea Surface, Wave Dynamics and Prediction, edited by A. Favre and K. Hasselmann, pp. 185-197, Plenum, New York, 1978.

Ling, S. C., T. W. Kao, M. Asce, and A. Saad, Microdroplets and transport of moisture from the ocean, J. Eng. Mech. Div. Am. Soc. Civ. Eng., 6, 1327-1339, 1980.

Meek, C. C., and B. G. Jones, Studies of the behavior of heavy particles in a turbulent flow, J. Atmos. Sci., 30, 239-244, 1973. 
Mestayer, P. G., De la structure fine des champs turbulents dynamique et thermique pleinement développés en couche limite, Thèse de Doctoratès-Sci. Phys., Univ. d'AixMarseille II, Marseille, France, 1980.

Mestayer, P. G., et al., CLUSE simulations of vapor flux transformations by droplet evaporation, in Modelling the Fate and Influence of Marine Spray, edited by P. G. Mestayer, E. C. Monahan, and P. A. Beetham, Whitecap Rep. 7, pp. 100-105, Mar. Sci. Inst., Univ. of Conn., Groton, 1990.

Mostafa, A. A., and H. C. Mongia, On the modeling of turbulent evaporating sprays: Eulerian versus Lagrangian approach, Int. J. Heat Mass Transfer, 12, 2585-2593, 1987.

Rouault, M. P., P. G. Mestayer, and R. Schiestel, A model of evaporating spray droplet dispersion, J. Geophys. Res., 96, 7181-7200, 1991.

Selva, J.-P., Etude de l'influence de la stratification sur l'evapo ration, Thèse de Doctorat de Eroisiemc Cycle, 173 pp., Inst. de Mec. Statist. de la Turbulance, Univ. d'Aix-Marseille II, Marseille, France, 1979.

Sini, J.-F., Modelisation d'ecoulements turbulents libres bidimensionnels avec effects de flottabilité. Thèse de Doctorat de 3 me Cycle, 285 pp., Inst. de Mec. Statist. de la Turbulence, Univ. d'Aix-Marseille II, Marseille, France, 1986.

Sini, J.-F., and I. Dekeyser, Numerical prediction of turbulent plane jets and forced plumes by use of the k-e model of turbulence, Int. J. Heat Mass Transfer, 30, 1787-1801, 1987.
Snyder, W. H., and J. L. Lumley, Some measurements of particle velocity autocorrelation functions in a turbulent flow, J. Fluid Mech., 48, 41-71, 1971.

Stramska, M., Vertical profiles of sea-salt aerosol in the atmospheric surface layer: A numerical model, Acta Geophys. Pol., 35, 87-100, 1987.

Stull, R. B., An Introduction to Boundary Layer Meteorology, 666 pp., Kluwer Academic, Norwell, Mass., 1988.

Wells, M. R., and D. E. Stock, The effects of crossing trajectories on the dispersion of particles in a turbulent flow, $J$. Fluid Mech., 136, 31-62, 1983.

$\mathrm{Wu}, \mathrm{J}$., Vertical distributions of spray droplets near the sea surface: Influence of jet drop ejection and surface tearing, J. Geophys. Res., 95, 9775-9778, 1990.

S. Anquetin, Laboratoire des Ecoulements Géophysiques et Industriels, Universite Joseph Fourrier, Grenoble, France.

(email: anquetin@img.fr)

J. B. Edson, Department of Applied Ocean Physics and Engineering, Woods Hole Oceanographic Institution, Woods Hole, MA 02543. (e-mail: jedson@airsea2.whoi.edu)

P. G.Mestayer and J. F. Sini, Laboratoire de Méchanique des Fluides, Ecole Centrale de Nantes, Nantes, France.

(e-mail: mestayer@ecnol.ec-nantes.fr)

(Received April 10, 1995; revised July 12, 1995; accepted July 21, 1995.) 